\title{
Journées scientifiques, Augsburg, septembre 1987
}

L'essentiel de ce cahier est consacré aux 23es Journées Scientifiques de la Société allemande de médecine sociale et de prévention. Ces journées scientifiques ont eu lieu en septembre 1987 à Augsburg et ont été consacrées aux thèmes suivants: «Les maladies cardiovasculaires - une tâche médicale et sociale».

De nombreux collègues suisses ont participé à ces journées, d'une façon très substantielle. L'un des faits marquants de cette journée a d'ailleurs été la remise de la médaille Salomon Neumann au Professeur F. H. Epstein (Zurich) pour sa contribution majeure à l'épidémiologie et à la prévention des maladies cardiovasculaires.

Le thème des maladies cardiovasculaires a été choisi parce que cette pathologie occupe une place particulière en République Fédérale Allemande. Chacun sait que la moitié des décès est attribuable à ce groupe de maladies. Si l'on considère simplement les décès prématurés enregistrés chez les hommes entre 40 et 69 ans, on constate que les maladies cardiovasculaires représentent $42 \%$ de la mortalité dans ce groupe. Les proportions correspondantes pour les cardiopathies ischémiques sont de $25 \%$. Les chiffres concernant les femmes sont moins impressionnants.

L'importance des maladies cardiovasculaires pour l'individu et pour la société est encore mieux perçu si, audelà de la mortalité, on considère la morbidité et l'invalidité associées à ces maladies. En République Fédérale Allemande et en 1984, la proportion des rentes pour incapacité professionnelle attribuable aux maladies cardiovasculaires est estimée à $35 \%$. Cette proportion concerne les hommes et les femmes, les employeurs et les employés. Les motifs d'attribution de rentes sont en premier lieu les cardiopathies ischémiques; environ un tiers des rentes ont été accordées avant l'âge de 54 ans.

Ces quelques chiffres illustrent clairement l'impact majeur des maladies cardiovasculaires sur la mortalité générale, la mortalité précoce, la morbidité et l'invalidité dans la population.
La prévention des maladies cardiovasculaires n'est pas une tâche exclusivement médicale, mais une tâche de la société. En République Fédérale Allemande, les efforts de prévention concernant un style de vie plus sain de la population se heurtent à de fortes oppositions. Presque chacune des modifications proposées entre en conflit avec les intérêts de certains groupes. Je ne pense pas ici seulement à l'industrie des cigarettes, mais également à l'agriculture allemande, qui cherche à mettre en doute les résultats de la «Lipid Research Clinics Coronary Primary Prevention Trial» dans les journaux médicaux, et à nier le lien causal entre la baisse du cholestérol alimentaire et celle de la mortalité par infarctus du myocarde.

Ces exemples montrent que les efforts à faire en République Fédérale Allemande sont encore énormes pour implanter les résultats d'importantes études épidémiologiques dans la pratique et pour le bien de la population.

Durant les journées scientifiques d'Augsburg, plus de 100 communications et posters ont été présentés sur l'épidémiologie, la prévention et la rééducation des maladies cardiovasculaires, ainsi que sur l'hypertension. Le projet de l'Organisation Mondiale de la Santé «MONICA» a également été dûment présenté. Des personnalités scientifiques de premier plan ont également participé dans les domaines de la physiopathologie, du diagnostic et du traitement des maladies coronariennes. Une séance parallèle des journées d'Augsburg a également abordé des aspects en-dehors du domaine des maladies cardiovasculaires.

Dans ce cahier, seule une partie des présentations et des posters ont été publiés. D'autres contributions pourront être adressées à la Rédaction pour publication, où elles suivront le processus habituel d'expertise.

Nous espérons que le présent numéro offre une image adéquate des Journées Scientifiques d'Augsburg, même si l'ensemble de toutes les contributions n'a pas pu être publié, pour des raisons de place et de temps.

Ulrich Keil 